\title{
NOT A S
}

\section{PRIMER REGISTRO DE LEPTODACTYLUS DIPTYX (Boettger, 1885), PARA EL DEPARTAMENTO ITUZAINGO, PROVINCIA DE CORRIENTES, ARGENTINA.}

Lisandro CARDINALE ${ }^{(1)}$; Nestor FARINA(2); Fernando BERNASCONI ${ }^{(1)}$ y Olga VILLALBA(2)

Desde el mes de enero de 2010, se llevan a cabo relevamientos y monitoreos de anfibios en la Reserva Natural Provincial Rincón Santa María y sus alrededores. La misma se encuentra ubicada al nordeste de la provincia de Corrientes, Argentina, aproximadamente a $12 \mathrm{~km}$ de la ciudad de Ituzaingó.

Durante la exploración de un nuevo sector distante a $6 \mathrm{~km}$ al norte de la Reserva y ubicado dentro del área de protección de la Central Hidroeléctrica de Yacyretá, sector de esclusa $\left(27^{\circ} 29^{\prime} 50,60^{\prime \prime} \mathrm{S} ; 56^{\circ} 42^{\prime} 12,13^{\prime}\right.$ 'O), se detectó la presencia de al menos cuatro ejemplares de Leptodactylus diptyx, que se encontraban vocalizando en un pastizal con sectores inundados por lluvias, sobre un sustrato de canto rodado.

La categorización más reciente del estado de conservación de las especies de anfibios de Argentina, considera a Leptodactylus diptyx como especie No Amenazada (Vaira et al., 2012) y se incluye como especie con Preocupación Menor a nivel global en la lista roja de especies amenazadas de la IUCN (IUCN, 2013). Su distribución en Argentina abarca las provincias de Corrientes, Chaco, Entre Ríos, Formosa, Santa Fe y Misiones. También está presente en Paraguay; Bolivia y Brasil (Frost, 2014). La especie ha sido registrada en 10 Departamentos de la provincia de Corrientes, con una distribución conocida que abarca desde el sur del Departamento de Bella Vista al oeste del Departamento General Paz (Zaracho et al., 2012).

El objetivo de esta nota es comunicar el hallazgo de un ejemplar colectado la noche del 16 de octubre de 2013 que amplía la distribución geográfica de Leptodactylus diptyx unos $60 \mathrm{~km}$ hacia el Este de su distribución conocida, extendiendo su rango hasta el Departamento Ituzaingó, Provincia de Corrientes, Argentina.

El ejemplar de referencia se encuentra depositado en la Colección Herpetológica de la Universidad Nacional del Nordeste, Corrientes, Argentina.

Leptodactylus diptyx (Boettger, 1885) (Anura, Leptodactylidae). UNNEC 12331 macho adulto, LHC $25 \mathrm{~mm}$.

(1) Entidad Binacional Yacyretá. Sector Medio Ambiente. Ituzaingó. Corrientes, Argentina.

(2) Reserva Natural Provincial Rincón Santa María. Ituzaingó; Corrientes; Argentina. Dirección de Parques y Reservas de la Provincia de Corrientes 


\section{BIBLIOGRAFÍA}

IUCN 2013. IUCN Red List of Threatened Species. Version 2013.2. www.iucnredlist.org. Consultada el 18 de diciembre de 2013.

Vaira, M.; M. Akmentins; M. Attademo; D. Baldo; D. Barrasso; S. Barrionuevo; N. Basso; B. Blotto; S. Cairo; R. Cajade; J. Céspedez; V. Corbalán; P. Chilote; M. Duré; C. Falcione; D. Ferraro; F.R. Gutierrez; M. Ingaramo; C. Junges; R. Lajmanovich; J.N. Lescano; F. Marangoni; L. Martinazzo; R. Marti; L. Moreno; G.S. Natale; J.M. Pérez Iglesias; P. Peltzer; L. Quiroga; S. Rosset; E. Sanabria; L. Sanchez; E. Schaefer; C. Ubeda y V. ZaRACHO, 2012. Categorización del estado de conservación de los anfibios de la República Argentina. Cuad. Herpetol., 26 (Supl.1): 131-159.

Frost, D.R. 2014. Especies de Anfibios del Mundo: una referencia en línea. Versión 6.0. (18 de junio de 2014). Museo de Americano de Historia Natural Nueva York. USA.

Zaracho, V.H; J.A. Céspedez; B.B. Alvarez y E.O. Lavilla, 2012. Guía de Campo Para la Identificación de los Anfibios de la Provincia de Corrientes, Argentina. $1^{\circ}$ Edición-Tucumán: Fundación Miguel Lillo 182 Pag.: il.; $25 \times 18 \mathrm{~cm}$. 